\title{
The ionosphere detector receiver channel fault analysis
}

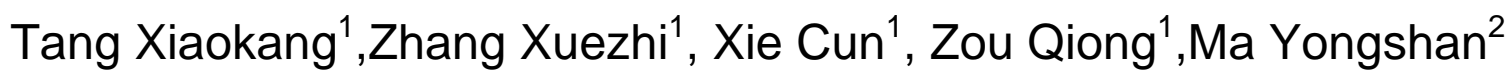

\section{Wuhan Mechanical Technology Colledge, Wuhan, China 2.The 65370 \\ Department,Changchun, China}

\begin{abstract}
Key words: the ionosphere detector; The receiver; Fault diagnosis; Analog channel
\end{abstract}
Abstract: in this paper, the ionospheric detector receiver analog channel are analyzed, and comb out the different kinds of common failures, due to the fault has the uncertainty and inaccuracy of how to modeling the ionosphere detector fault reasoning, and how to the ionosphere detector is a key problem for its fault diagnosis.

\section{Summary of the receiving system analog channel}

Isolation protection switch: the ionosphere detectors in the vertical and inclined to return the single station detection, belong to the single base radar, send and receive two antennas in the same site, the radar's transmitted power is larger, according to different detection target and demand, transmitted power can be reached from a few hundred watts supreme kw. High power of radar signal in the radar station form a strong local near field signal is referred to as the "ground" [33]. This kind of ground wave transmitting directly reflect coupling or after nearly ground coupled to the strength of the receiving antenna signal. High power ground wave signal directly into the receiver will cause the receiver when overload, even damage the receiver, affect the normal reception of the echo.

Using pulse phase modulation system of monostatic radar due to the pulse sequence is sent, the transmitted pulse, cut off, the switch to prevent the ground wave signal directly into the receiver, receiver overload. The radar pulse phase modulation system in order to obtain high range resolution, launched by the pulse width is narrower. Not only reduces the narrow pulse transmitting power, also require switch have higher switching speed in order to make sure when the transmitted pulse switch off, when firing pulse interval pulse open [34]. Adoption of pulse phase modulation between detectmonostatic radar, due to switch off and blind spot detection, if switch slower will increase the speed of detection blind area [35]. If the radar to obtain the resolution in range of $7.68 \mathrm{~km}$, a single pulse width is 51.2 us, This request to receive the front switch conduction and shut off state switch time is less than 1 us to ensure that the receiver will not overload, blind area will not be expanded.

Low pass, high pass module: this module is responsible for the antenna signal filtering, to receive signals frequencies within 1.6-30 m only less than $1 \mathrm{db}$ attenuation, the outside of the receiving frequency signal for 30 to $60 \mathrm{db}$ attenuation, secondly on the vibration signal (43-71.4 $\mathrm{MHz}$ ) leakage signal can also be suppressed, to improve the medium frequency rejection ratio and mirror frequency rejection ratio, suppress clutter interference has a very important role [36]. At the 
same time, due to below $1.6 \mathrm{MHz}$ is stronger in the long wave interference signal, the filter can effectively filter module.

Second mixer, 2 vibration wide and 2 matching amplifier: the second mixer is mainly composed of passive mixer component and low-pass filter circuit, an intermediate frequency signal is sent to and from $40 \mathrm{MHz}$ frequency synthesizer unit 2 vibration signals into the mixer, the frequency of $1.4 \mathrm{MHz} 2$ of intermediate frequency signal by the LC low pass filter filter and matching.

\section{Process analog channel receiver to work normally}

Preparation: link good power supply (ac 220 v), RF, RF, RF input RF output after make sure wire, USB, GPS control bus to work on electricity;

Boot: through to the machine at the back of the ionosphere detector switch, can hear the sound of the fan rotation, all green lights on the panel light;

Work: turn on the panel on the standby \ work key, when pressed in a working state, locking frequency indicator, PPS indicator, said received GPS clock signal. Echo signal lights that channel analog part of the received echo signal receiver; If the button is not bottom go to, receiver is in standby state, even with rf signal input, also won't send signals to the signal processing part of the display.

Monitoring: the ionosphere on the instrument panel can monitor to the receiver channel state and fault information. Each module has the supply, the power indicator light is green. Each amplifier module has rf lamp, can be intuitive instructions including two levels of mixer, two-stage amplifier signal output.Ionospheric frequency precision instrument panel lights can also monitor to the GPS signal. When the panel "switch" mixing 1 "protect" enlarge "1" "mixing 2" enlarge "2" and so on the corresponding red indicator, respectively corresponding fault, $\mathrm{rf}$ input signal is received at this time the opportunity to cut off for their own protection.

\section{The receiver analog channel common faults}

Fault phenomenon one: the ionosphere detectors after startup, launch a functional fire signals into space, no echo signal display.

Fault phenomenon one: the ionosphere detectors after startup, launch a functional fire signals into space, no echo signal display.

Serious fault phenomenon. 2: clutter interference, floods the echo.

This fault should be out in the receiving channel analog part, check the isolation protection switch, low-pass qualcomm module.

Three failure phenomenon: receives the echo weak sometimes no. 
This fault should be out in the receiving system analog channel power amplification device, device leads to aging or failure.

Case 4: "protection switch" indicator, caused by protection switch is not normal. First high detection protection switch $+8 \mathrm{v}$ power supply voltage is normal, please check if normal connection cable no intact; If the power supply box and cable is good, will replace the transceiver protection switch. When sending and receiving protection switch failure of the ionosphere detectors will enter a state of protection, stop firing rf signal.

Case 5: "mixing 1" indicator, analog channel receiver first mixer can't work normally. The first detection filter output signal is normal; The second check $44.474 .4 \mathrm{MHz}$ this vibration signal is normal; The third if filter and the local oscillator signal is normal, could be replaced damaged mixer transistor mixing transistor.

Case 6: "enlarge 1" indicator, the first level high frequency amplifier doesn't work. First check whether the gating switch is normal if normal examined for gating switch control signal, if you have normal high-frequency amplifier and switch signal input signal is too small. If are in good condition and good contact, check the $12 \mathrm{v}$ working voltage amplifier is normal, if abnormal voltage change of power supply module.

Case 7: "mixing 2" indicator, the second mixer channel receiver simulation can't work normally. The first detection filter output signal is normal; The second check 44.4 74.4 MHz this vibration signal is normal; The third if filter and the local oscillator signal is normal, could be replaced damaged mixer transistor mixing transistor.

Case 8: "enlarge 2" indicator, the second level high frequency amplifier doesn't work. First check whether the gating switch is normal if normal examined for gating switch control signal, if you have normal high-frequency amplifier and switch signal input signal is too small. If are in good condition and good contact, check the $12 \mathrm{v}$ working voltage amplifier is normal, if abnormal voltage change of power supply module.

Case 9: state of the ionosphere detector lamp 1, lamp 2, and 3 normal, instructions, other state light is not bright. Illustrate the ionosphere detector AGC intermediate frequency amplifier in normal and fault in gating switch and the second between intermediate frequency amplifier output to the $\mathrm{A} / \mathrm{D}$ converter. As

Fault rule set is as follows:

IF AGC echo signal THEN gating switch output signal $\mathrm{CF}=0.96$;

IF the clock signal THEN gating switch output signal CF $=0.94$;

IF gating switch signal THEN mixer output signal CF $=0.93$;

IF the local oscillator signal THEN CF $=0.95$ mixer output signal;

IF lose output signal mixer THEN lights work $456 \mathrm{CF}=0.92$; 
Based on the fuzzy Petri net said method of production rules, these rules can be converted to fuzzy Petri net model, as shown in figure1. P1: for AGC echo signal; P2: the clock signal; P3: gating switch output signal; P4: mixer output signal; P5: the local oscillator signal; The p6 light 456 working condition.

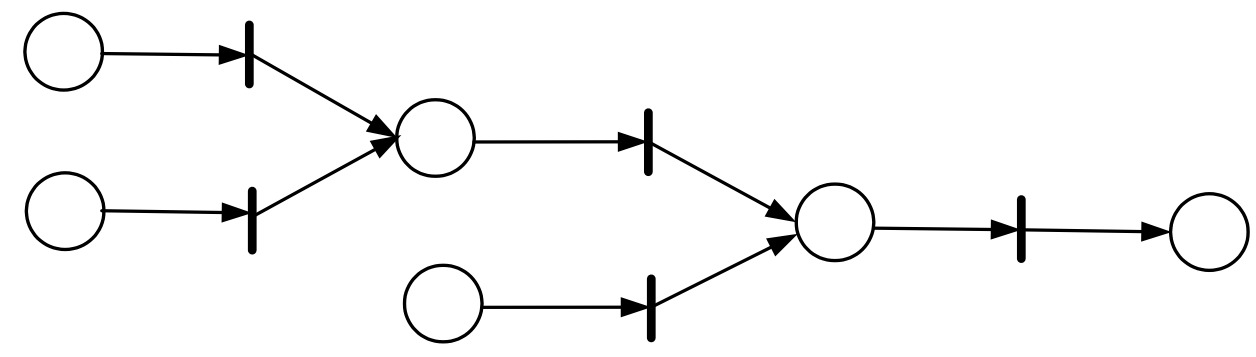

Figure1 indicator 456 not on Petri net model

If the anomalies (credibility of 0.99), the vibration signal is abnormal propagation path is shown in figure2

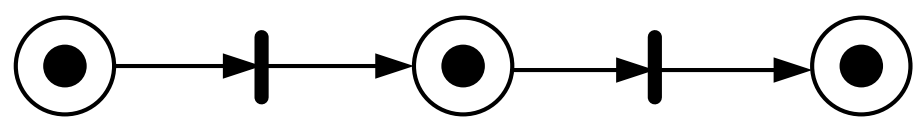

Figure2 exception propagation path

The exception propagation path can be calculated by the credibility of the final state light does not work for: $0.99 * 0.95 * 0.95=0.865>0.7$ (trigger threshold), will cause the status indicator light does not work. According to this chapter introduces reverse reasoning algorithm of fuzzy Petri nets, first of all, according to the size of the Transition confidence, by selecting the confidence the biggest Transition, determine the most likely reason for $\mathrm{P} 4$, the vibration signal failure. If the user input its credibility is 0.9 , forward reasoning, can judge the reason to cause failure indicator 456 not bright. Can calculate its credibility is: $0.9 * 0.95 * 0.95=0.7866>0.7$, reason can cause this failure, the $\mathrm{P} 4$ for root cause.

\section{References}

[1] Ma Jian-mei. Environmental art course teaching mode reform of professional landscape design [J]. Anhui Agricultural Science. 2011 (31)

[2] Tang Jun. The development of modern landscape design present situation and trend analysis [J]. Chinese Gardening Abstract. 2012 (9) : 97-2012.

[3] Han Yu. Experience type teaching, the landscape design course teaching practice research [D]. Nanjing: Nanjing College of Art. 2012

[4] Xiang Xiaoxiao. Environmental art professional teaching reform of landscape design study [J]. Journal of Nanchang Institute of Education. 2013 (4)

[5] liu Chun-bo . Analyses of landscape environmental art [J]. The Young Writer. 2010 (18) 